\title{
Avaliação de uma Capacitação para Profissionais de Psicologia que Trabalham com Vítimas de Abuso Sexual
}

\author{
Clarissa Pinto Pizarro de Freitas \\ Universidade Federal do Rio Grande do Sul \\ Porto Alegre, RS, Brasil \\ Luísa Fernanda Habigzang \\ Pontificia Universidade Católica do Rio Grande do Sul \\ Porto Alegre, RS, Brasil \\ Sílvia Helena Koller \\ Universidade Federal do Rio Grande do Sul \\ Porto Alegre, RS, Brasil
}

\begin{abstract}
RESUMO
O presente estudo objetivou avaliar a efetividade de uma Tecnologia Social de Capacitação Profissional para intervenção com vítimas de abuso sexual (TSCP-AS) por meio dos níveis de impacto e transferência de aprendizagem. Participaram da pesquisa 32 profissionais de psicologia (94\% do sexo feminino), com idade média de 35,5 anos $(\mathrm{DP}=10,7)$. Os níveis de impacto e transferência de aprendizagem foram avaliados em dois momentos, ao término dos módulos instrucionais e após a conclusão da TSCP-AS. Foram observados altos níveis de impacto e transferência de aprendizagem no primeiro e segundo momento de avaliação. Estes achados sugerem que a TSCP-AS constitui-se como uma intervenção efetiva à qualificação dos profissionais de psicologia para o atendimento de vítimas de abuso sexual. Além disso, foi demonstrado que os níveis de impacto podem predizer os índices de transferência de aprendizagem, assim como a transferência de aprendizagem prediz o impacto.
\end{abstract}

Palavras-chave: Formação profissional; Impacto; Transferência de aprendizagem; Abuso sexual.

\begin{abstract}
Evaluation of a Training for Psychologists Working with Victims of Sexual Abuse

This study aimed to evaluate the effectiveness of a Social Technology of Professional Training for an Intervention involving victims of sexual abuse (STPT-SA) by assessing the impact and learning transfer levels. Thirty-two psychologists $(94 \%$ female) participated in this research, with a mean age of 35.5 years $(\mathrm{SD}=10.7)$. The impact and learning transfer levels were evaluated at two moments, at the end of instructional modules and after the end of the STPT-SA. High levels of impact and learning transfer were observed in the first and second assessments. These findings suggest that STPT-SA is an effective intervention for professional training of psychologists who provide care to victims of sexual violence. Furthermore, it was observed that levels of impact can predict learning transfer rates, in the same way as the learning transfer predicts impact.
\end{abstract}

Keywords: Professional education; Impact; Transfer of learning; Sexual abuse.

\section{RESUMEN}

Evaluación de una Capacitación para Profesionales Psicología que Trabajan con Víctimas de Abuso Sexual

El presente estudio tuvo como objetivo evaluar la eficacia de una Tecnología Social de Capacitación Profesional para la Intervención con Víctimas de Abuso Sexual (TSCP-AS) a través de los niveles de impacto y la transferencia del aprendizaje. Participaron en la investigación 32 profesionales en psicología (94\% mujeres) con una edad media de 35,5 años $(D E=10,7)$. Los niveles de impacto y transferencia de aprendizaje se evaluaron en dos momentos, en la finalización de los módulos de instrucción y al término de la TSCP-AS. Se observaron altos niveles de impacto y transferencia de aprendizaje en el primero y el segundo momento de evaluación. Estos hallazgos sugieren que la TSCP-AS constituye como una intervención efectiva para la cualificación de los profesionales de psicología para atender a las víctimas de abuso sexual. Además, se observó que los niveles de impacto pueden predecir los índices de transferencia de aprendizaje, así como la transferencia de aprendizaje predice el impacto.

Palabras clave: Formación profesional; Impacto; Transferencia de aprendizaje; Abuso sexual. 
Os avanços a proteção integral às crianças e aos adolescentes no Brasil possibilitaram que o atendimento eficaz e imediato das vítimas de abuso sexual (AS) após a revelação dessa violência fosse estabelecido como um direito fundamental, conforme instituído pela Lei n. 8069/1990 do Estatuto da Criança e do Adolescente (ECA; Brasil, 1990). Entretanto, a realidade observada em diferentes contextos indica que os serviços de proteção às crianças e aos adolescentes são, por vezes, incapazes de fornecer atendimento adequado por lacunas em sua formação profissional (Conselho Federal de Psicologia [CFP], 2009; Habigzang, Ramos, \& Koller, 2011; Luna, Ferreira, \& Vieira, 2010). Entre as dificuldades enfrentadas pelos profissionais de psicologia no atendimento às vítimas, aquelas que têm sido frequentemente identificadas são: o desconhecimento de tratamentos baseados em evidências; inabilidade técnica; desconhecimento da dinâmica do abuso sexual (Freitas \& Habigzang, 2014; Saywitz, Mannarino, Berliner, \& Cohen, 2000); a incapacidade dos profissionais identificarem sintomas em crianças e adolescentes que indiquem a suspeita da ocorrência de AS (Gonçalves \& Ferreira, 2002; WHO, 2006); assim como dificuldades em realizar denúncias, em razão das implicações legais e éticas (Saywitz et al., 2000).

Em razão do impacto negativo do AS ao desenvolvimento das vítimas (Chen et al., 2010; Maniglio, 2009), os profissionais de psicologia são orientados a utilizarem tratamentos baseados em evidências (TBE) no atendimento de crianças e adolescentes que foram expostos a esta violência (Sanson, 2011; World Health Organization [WHO], 2006). Entretanto, os TBE ainda não estão significativamente integrados nas políticas públicas de proteção às crianças e adolescentes vítimas de AS (WHO, 2006). Diferentes fatores são indicados como obstáculos à aplicação de TBE, entre estes a ausência de formação adequada, a sobrecarga de trabalho, o desconhecimento do tema (Freitas \& Habigzang, 2013; Luna et al., 2010; Souza, McCarthy, \& Gauer, 2012). Outras dificuldades que podem estar presentes são a inabilidade técnica dos profissionais para atenderem as demandas relacionadas ao AS (Saywitz, Mannarino, Berliner, \& Cohen, 2000), a ausência de apoio para os profissionais lidarem com demandas de trabalho emocionalmente exaustivas (Shapiro, Dorman, Burkey, \& Welker, 1999) e as condições de trabalho precárias (Santos, Pereira, \& Carlotto, 2010).

A efetividade dos tratamentos psicoterápicos para crianças e adolescentes vítimas de AS tem sido investigada, a fim de disponibilizar as melhores estratégias de tratamento (Cohen, Deblinger,
Mannarino, \& Steer, 2004; Cohen, Mannarino, \& Knudsen, 2005; Habigzang et al., 2009; Habigzang, Damásio, \& Koller, 2013; Hetzel-Riggin, Brausch, \& Montgomery, 2007). Entre os tratamentos desenvolvidos para o atendimento de vítimas de AS, foi observado que os pacientes que receberam o tratamento baseado na terapia cognitivo-comportamental focada no trauma apresentaram melhoras significativas em relação: aos sintomas relacionados ao TEPT; aos sintomas depressivos; aos problemas de comportamento; à confiança interpessoal; às percepções de que recebe credibilidade; aos sentimentos de vergonha; e às atribuições distorcidas relacionadas ao AS (Cohen et al., 2004). Além disso, o tratamento baseado na terapia cognitivo-comportamental focado no trauma para vítimas de AS promoveu uma redução e manutenção das melhoras terapêuticas em seis e 12 meses nos sintomas relacionados ao TEPT, especialmente nos sintomas dissociativos, sintomas depressivos, sintomas de ansiedade, e nos comportamentos hipersexualizados (Cohen et al., 2005).

No Brasil, tem sido evidenciada a efetividade do Programa SUPERAR (Habigzang, Damásio, \& Koller, 2013; Habigzang et al., 2009). Este programa consiste em uma intervenção cognitivo-comportamental breve e estruturada, a qual demonstrou ser efetiva ao tratamento de meninas na modalidade grupal (Habigazng et al., 2013; Habigzang et al., 2009), assim como no atendimento de meninos na modalidade individual (Hohendorff, 2012).

O modelo de grupoterapia cognitivo-comportamental para vítimas de AS do Programa SUPERAR constitui-se por 16 sessões com frequência semanal, totalizando quatro meses de duração. As sessões são divididas em três etapas, sendo a primeira 'Psicoeducação', constituída por seis encontros, a segunda 'Treino de Inoculação do Estresse', composta por quatro sessões, e a 'Prevenção à Recaída', que compreende as últimas seis sessões da intervenção (Habigzang et al., 2013; Habigzang et al., 2009). Este programa demonstrou ser efetivo na redução significativa de sintomas de depressão, ansiedade, estresse e TEPT, além de contribuir para a redução de crenças distorcidas relacionadas à culpa pela AS, confiança nas outras pessoas e credibilidade (Habigzang et al., 2009). Além disso, a melhora dos sintomas de ansiedade, estresse e TEPT foi mantida por seis e 12 meses após o término do tratamento (Habigzang et al., 2013).

A estrutura do Programa SUPERAR na modalidade de atendimento individual possui uma estrutura semelhante à presente na grupoterapia cognitivocomportamental (Hohendorff, 2012). Em razão dos 
mitos sociais e os estereótipos relacionados ao gênero masculino, tal como "meninos não são vítimas de abuso sexual", foram incluídos temas específicos à vitimização masculina (Hohendorff, Habigzang, \& Koller, in press). Desta forma, ao longo do processo terapêutico foram realizadas discussões sobre orientação sexual e identidade de gênero (Hohendorff et al., in press). A inclusão das discussões sobre estas temáticas na realização desta intervenção foi devido às evidências de que as principais dificuldades das vítimas do sexo masculino estão relacionadas à sua masculinidade e orientação sexual (Alaggia, 2005; Hohendorff, 2012; Lisak, 1994).

As evidências de efetividade do Programa SUPERAR no contexto brasileiro (Habigzang, Damásio, \& Koller, 2013), associado à relevância dos profissionais de psicologia utilizarem TBE (Sanson, 2011; WHO, 2006), motivou o desenvolvimento de uma Tecnologia Social de Capacitação Profissional para intervenção com vítimas de abuso sexual (TSCPAS; Freitas \& Habigzang, 2014). As temáticas trabalhadas na TSCP-AS abarcaram a definição dos maus-tratos contra crianças e adolescentes, dinâmica do AS, consequências ao desenvolvimento da vítima, as intervenções realizadas pelos profissionais de psicologia ao identificarem casos de suspeita ou confirmação de AS, e modelo de avaliação psicológica às vítimas (Freitas \& Habigzang, 2014). Além disso, buscou instrumentalizar os profissionais para aplicação do Programa SUPERAR, discutindo o modelo de grupoterapia cognitivo-comportamental para meninas de AS e a modalidade de atendimento individual para meninos vítimas (Habigzang et al., 2013; Habigzang et al., 2009; Hohendorff, 2012).

A TSCP-AS foi composta por uma etapa instrucional e uma etapa teórico-prática. A etapa instrucional foi desenvolvida ao longo dos três primeiros módulos. A etapa teórico-prática, por sua vez, constitui o quarto módulo. Neste módulo final foram desenvolvidas cinco supervisões dos atendimentos clínicos realizados no modelo de grupoterapia para crianças e adolescentes vítimas. A carga horária total foi de 34 horas, sendo 24 horas referentes à parte instrucional da capacitação e 10 horas às supervisões dos atendimentos (Freitas \& Habigzang, 2014). A fim de potencializar o aprendizado do modelo de grupoterapia cognitivocomportamental, os participantes receberam o livro "Intervenção psicológica para crianças e adolescentes vítimas de violência sexual - Manual de capacitação profissional" (Habigzang \& Koller, 2011), desenvolvido especificamente para a TSCP-AS, uma vez que foi observado que os manuais auxiliam os profissionais na aplicação de TBE (Kendall \& Beidas, 2007;
WHO, 2006). Além deste, foram sugeridos materiais científicos que abordavam os temas relacionados aos conteúdos dos módulos teórico-práticos para estudo (Freitas \& Habigzang, 2014).

As evidências de efetividade de capacitações à qualificação profissional têm indicado que maiores índices de impacto e transferência de aprendizagem estão associados à utilização intervenções com delineamento estruturado e a realização de mais de um encontro (Aguinis \& Kraiger, 2009; Grossman \& Salas, 2011; Rakovshik \& McManus, 2010). Foi observado também que as capacitações à qualificação profissional podem apresentar maior efetividade quando utilizam manuais sobre a intervenção e são adaptadas às características contextuais dos participantes (Kendall \& Beidas, 2007). Além disso, o desenvolvimento de supervisões da aplicação das técnicas trabalhadas pelos participantes constitui-se como um elemento essencial na promoção do impacto e transferência de aprendizagem na atuação profissional deles (Clarke, 2002; Rakovshik \& McManus, 2010).

Tendo em vista a necessidade de serem realizadas capacitações que promovam a qualificação profissional, avaliou-se a efetividade da TSCP-AS como um recurso para instrumentalizar os profissionais de psicologia ao atendimento das demandas relacionadas ao AS e na aplicação do Programa SUPERAR. A avaliação de efetividade foi realizada por meio da mensuração dos níveis de impacto e transferência de aprendizagem, pois essas evidenciam se os profissionais estão utilizando os conteúdos, as técnicas e as habilidades aprendidas na capacitação em seu contexto de trabalho (Aguinis \& Kraiger, 2009; Grossman \& Salas, 2011).

O impacto no trabalho caracteriza-se pelas repercussões da capacitação sobre o desempenho e envolvimento dos profissionais com suas atividades laborais. Os efeitos do impacto podem não estar diretamente relacionados aos objetivos da capacitação, os quais geralmente englobam o aumento da motivação, de autonomia e uso de habilidades, e, consequentemente, melhoria no desempenho laboral e relacionamento interpessoal (Abbad, Gama, \& BorgesAndrade, 2000; Pilati \& Abbad, 2005). A avaliação de impacto analisa o resultado final de um processo que se inicia com a inserção dos profissionais na capacitação e alcança seu ápice no retorno deles ao seu ambiente laboral. Este processo está relacionado aos processos de aquisição, retenção, generalização e transferência dos conteúdos, técnicas e habilidades abordados, os quais são essenciais para a ocorrência de impacto (Pilati \& Abbad, 2005; Zerbini \& Abbad, 2010).

A aquisição caracteriza-se como um processo de aprendizagem dos participantes dos conteúdos e 
técnicas trabalhados na capacitação. Posteriormente a esse processo, identifica-se a retenção: esta se refere à compreensão e resignificação pelos profissionais das temáticas trabalhadas, de modo que seja possível a eles armazenarem estas informações na memória de longo prazo. A generalização constitui-se como uma habilidade desenvolvida no processo de qualificação profissional, na qual os participantes tornam-se capazes de reconhecerem as diferentes oportunidades em seu contexto laboral em que eles podem utilizar as habilidades e técnicas aprendidas (Pilati \& Abbad, 2005). A transferência de aprendizagem, por sua vez, é reconhecida nas ações em que os profissionais aplicam de forma efetiva os conteúdos, técnicas e habilidades adquiridos (Taxman, Henderson, Young, \& Farrell, 2014). Para a transferência de aprendizagem ser identificada deve haver a utilização efetiva das habilidades aprendidas pelo profissional e a aplicação do conhecimento aprendido durante a capacitação deve permanecer ocorrendo após o término dessa (Cheng \& Ho, 2001; Zerbini \& Abbad, 2010).

Observa-se que a transferência de aprendizagem deve ser avaliada conjuntamente ao impacto do trabalho, pois esta evidencia se os participantes estão transmitindo efetivamente o conhecimento e as intervenções aprendidos na capacitação a sua prática profissional (Cheng \& Ho, 2001; Taxman et al., 2010). A transferência de aprendizagem pode ser classificada como direta, ou em curto prazo, e indireta, ou em longo prazo (Garavaglia, 1995). A transferência em curto prazo refere-se à utilização do conhecimento e técnicas aprendidas em situações semelhantes às trabalhadas durante o processo de qualificação profissional. A transferência em longo prazo caracteriza-se pela aplicação do conhecimento e intervenções em diferentes contextos, nos quais os profissionais identificam oportunidades diferentes das propostas na capacitação, nas quais habilidades desenvolvidas podem ser efetivamente aplicadas (Garavaglia, 1995).

O impacto e a transferência de aprendizagem constituem-se como repercussões de uma capacitação sobre o desempenho laboral dos profissionais em seu trabalho. (Abbad, Borges-Andrade, \& Sallorenzo, 2004; Zerbini \& Abbad, 2010). Estes eventos são fortemente relacionados e são multideterminados, sendo influenciados pela reação dos profissionais a intervenção, conhecimento prévio, níveis de autoeficácia ocupacional e satisfação no trabalho (Abbad et al., 2004; Cheng \& Ho, 2001; Garavaglia, 1995; Taxman et al., 2014). Em razão das relações entre estas dimensões, sugere-se que se pode formar uma espiral em que a presença de altos níveis de impacto pode aumentar os níveis de transferência de aprendizagem, assim como altos níveis de transferência de aprendizagem podem potencializar o impacto da capacitação sobre o trabalho ao longo do tempo.

Com base no exposto, observa-se que os níveis de impacto e transferência de aprendizagem constituem-se como indicadores que podem contribuir na construção de programas de qualificação profissional efetivos e relevantes ao contexto social (Rossi, Lipsey, \& Freeman, 2004). Desta forma, o presente estudo objetivou investigar os níveis de impacto e transferência de aprendizagem da TSCP-AS sobre o trabalho dos profissionais de psicologia que atendem crianças e adolescentes vítimas de AS. Além disso, foi avaliado se os níveis de impacto e transferência de aprendizagem formavam um espiral de crescimento, no qual os níveis de impacto e transferência de aprendizagem após a conclusão dos módulos instrucionais constituíamse como preditores, respectivamente, dos índices de transferência de aprendizagem e impacto após o término da TSCP-AS.

\section{MÉTODO}

\section{Participantes}

Participaram do estudo 32 profissionais de psicologia que trabalhavam em instituições públicas de atendimentos às vítimas de $\mathrm{AS}$ de 25 municípios do Estado do Rio Grande do Sul. A amostra foi formada predominantemente por mulheres (94\%), a idade média dos(as) participantes foi de 35,5 anos $(D P=10,7$ anos, amplitude 24 a 63 anos). Em relação às características laborais, o tempo de trabalho médio em instituições que atendem vítimas de abuso sexual variou de um mês a 12 anos e três meses. Entre os participantes, $50 \%$ dos(as) profissionais citaram ter ao menos uma pós-graduação, sendo que: $9 \%$ possuíam formação em terapia sistêmica; $6 \%$ possuíam formação em psicoterapia orientação analítica; $6 \%$ terapia cognitivocomportamental; $3 \%$ possuíam especialização em psicopedagogia; 3\% em avaliação psicológica; 3\% gestão de pessoas; 3\% violência doméstica; 3\% em análise institucional; 3\% psicologia escolar; e 3\% em saúde coletiva.

\section{Instrumentos}

Questionário Biossociodemográfico e Laboral: Este instrumento investigou informações biossociodemográficas e laborais, tais como gênero, idade, estado civil, escolaridade e tempo de trabalho.

Escala de Impacto do Treinamento no Trabalho (EIT): Esta escala foi desenvolvida e validada por Abbad (1999). Foi utilizada para investigar o impacto do treinamento por meio dos indicadores: melhora do 
desempenho no trabalho; motivação para realização das atividades ocupacionais; e atitude favorável à modificação da forma de se realizar o trabalho (Abbad, 1999). A escala possui uma estrutura unifatorial com alpha de Cronbach > 0,90 (Pilati \& Abbad, 2005). Na amostra estudada, a estrutura unifatorial da escala foi observada, sendo que o valor de alpha de Cronbach foi adequado $(\alpha=0,85)$.

Escala de Transferência de Aprendizagem (ETA): Esta escala objetivou avaliar os níveis de transferência de aprendizagem dos profissionais ao contexto de trabalho. Foram investigados os comportamentos dos profissionais que explicitam a aplicação do conhecimento, habilidades e técnicas adquiridas na capacitação. Desta forma, foi pedido que os profissionais indicassem com que frequência utilizavam os conteúdos e técnicas trabalhadas TSCP-AS no atendimento de suas demandas de trabalho. Os índices de transferência de aprendizagem foram avaliados para as demandas que envolviam situações de AS, assim como em situações que não envolviam situações de AS. Esta escala possui uma estrutura unidimensional e valor de consistência interna satisfatório $(\alpha=0,81)$ (Freitas, 2013).

\section{Procedimentos}

Esta pesquisa faz parte de um projeto maior intitulado "Avaliação de Programa de Capacitação Profissional e de Intervenção Psicológica para Crianças e Adolescentes Vítimas de Abuso Sexual". Este projeto teve como objetivo avaliar a aplicação do modelo de grupoterapia cognitivo-comportamental por profissionais da rede de atendimento público às crianças vítimas de AS, no estado do Rio Grande do Sul. A pesquisa foi aprovada pela Comissão de Ética e Pesquisa em Saúde do Grupo Hospitalar Conceição (CEP-GHC), número do protocolo: 00150164000-10.

A TSCP-AS foi realizada em oito encontros de frequência mensal. Os encontros foram divididos em quatro módulos, sendo que os três primeiros módulos referiam-se à parte instrucional, e o quarto módulo constituiu-se por cinco supervisões dos atendimentos clínicos realizados no modelo de grupoterapia para crianças e adolescentes vítimas. Os módulos instrucionais da TSCP-AS foram ministrados por profissionais de psicologia especialistas, estudantes de mestrado e doutorado, membros do CEP-Rua, com experiência clínica no atendimento e na pesquisa clínica com crianças e adolescentes vítimas de AS. Os recursos didáticos utilizados foram: aulas expositivodialogadas; apresentações de vídeos; discussão de casos clínicos; e dramatizações. As supervisões dos atendimentos clínicos foram realizadas por profissionais de psicologia com experiência clínica no atendimento e na pesquisa clínica com crianças e adolescentes vítimas de AS (Freitas \& Habigzang, 2014).

Ao serem convidados a contribuir com o estudo, os participantes receberam o Termo de Consentimento Livre e Esclarecido, explicando o objetivo do estudo e que sua participação era voluntária. A coleta de dados foi realizada de forma coletiva, em salas de aulas onde a TSCP-AS foi desenvolvida. O questionário sociodemográfico foi respondido no primeiro encontro. As escalas de impacto do treinamento e transferência de aprendizagem foram aplicadas em dois momentos, ao final do terceiro módulo, referente à conclusão da etapa instrucional da capacitação (T1), e após a conclusão do quarto módulo e término da TSCP-AS (T2). O intervalo de tempo entre $\mathrm{T} 1$ e $\mathrm{T} 2$ foi de cinco meses.

\section{Análise dos dados}

A normalidade da distribuição dos dados foi investigada por meio do teste de Kolmogorov-Smirnov e foram realizadas análises descritivas (medidas de tendência central e desvio padrão). Foi investigado se os níveis de impacto e transferência de aprendizagem formam um espiral de crescimento e, para isso, foram realizadas duas regressões lineares. $\mathrm{Na}$ primeira regressão foi testado se os índices de impacto em T1 predizem a transferência de aprendizagem em T2. Na segunda regressão, foi avaliado se a transferência de aprendizagem em T1 prediz o impacto em T2. Em razão dos pressupostos de normalidade da amostra terem sido violados, foi utilizada a técnica de bootstraping (2.000 reamostragens), com um intervalo de $99 \%$ de confiança da diferença da média (Haukoos \& Lewis, 2005).

\section{RESULTADOS}

A média dos níveis de impacto dos profissionais que participaram da capacitação em $\mathrm{T} 1$ foi de 4,40 $(D P=0,49)$. Em T2, pode-se observar que os escores de impacto mantiveram-se altos $(M=4,34 ; D P=0,58)$. As médias dos índices de transferência de aprendizagem dos participantes da capacitação foram altas em T1 $(M=4,42 ; D P=0,36)$ e em T2 $(M=4,11 ; D P=0,53)$.

A fim de investigar se as relações entre os níveis de impacto e transferência de aprendizagem formam uma espiral de crescimento, foram realizadas duas regressões lineares. Na primeira regressão os níveis de impacto em $\mathrm{T} 1$ foram estabelecidos como preditores dos índices de transferência de aprendizagem em T2. Na segunda, foi verificado se os níveis de transferência de aprendizagem em $\mathrm{T} 1$ predizem os níveis de impacto em T2. Os níveis de impacto em T1 explicaram 42\% dos índices de transferência de aprendizagem em 
T2. Foi observado que os níveis de transferência de aprendizagem em $\mathrm{T} 1$ contribuíram em $40 \%$ dos índices de impacto em T2 (Tabela 1).

TABELA 1

Regressões Lineares entre transferência de aprendizagem em T1 e impacto em T2 e impacto em T1 com transferência de aprendizagem em T2 $(\mathrm{N}=32)$

\begin{tabular}{lccc}
\hline \multicolumn{1}{c}{ Dimensão } & $r^{2} A j$. & $\begin{array}{c}\beta \\
(99 \% I C)\end{array}$ & $F$ \\
\hline Impacto do Treinamento (T2) & 0,42 & \multicolumn{2}{c}{$8,5^{*}$} \\
Transferência de Aprendizagem (T1) & & $\begin{array}{c}0,64^{*} \\
(0,18-0,73)\end{array}$ & \\
Transferência de Aprendizagem (T2) & 0,40 & & $9,7^{*}$ \\
Impacto do Treinamento (T1) & \multicolumn{3}{c}{$\begin{array}{c}0,67^{*} \\
(0,35-1,23)\end{array}$} \\
\hline
\end{tabular}

* $p \leq 0,05 ; * * p \leq 0,001$

\section{DISCUSSÃO}

Os altos níveis de impacto no primeiro momento da coleta, assim como a manutenção dos altos valores no segundo momento, sugerem que a TSCP-AS contribuiu na qualificação dos profissionais e no desenvolvimento do seu trabalho (Zerbini \& Abbad, 2010). Os resultados observados na avaliação do impacto e transferência de aprendizagem demonstram que os profissionais utilizaram os conteúdos e técnicas aprendidos durante a TSCP-AS. Além disso, estes resultados indicam que após a conclusão da TSCP-AS os profissionais apresentam um alto potencial de permanecerem aplicando os conteúdos e as técnicas trabalhados ao seu contexto laboral (Rodríguez \& Gregory, 2005).

Foram observados altos índices de transferência de aprendizagem em T1 e T2, os quais sugerem que os(as) participantes generalizaram os conteúdos e as técnicas trabalhados na TSCP-AS ao contexto de trabalho (Abbad et al., 2004; Taxman et al., 2014). Os altos índices de transferência de aprendizagem indicam que os profissionais podem ter modificado suas práticas na atuação profissional, tais como utilizar as técnicas de avaliação psicológica e realizar o atendimento as vítimas de AS segundo o modelo de grupoterapia cognitivo-comportamental. Corroborando estes achados, foi observado que ao final da TSCP-AS, foi realizado o atendimento a 26 vítimas de abuso sexual, sendo desenvolvidos seis grupos, por seis profissionais. Além disso, seis profissionais atenderam ao total 10 crianças (nove pacientes eram do sexo feminino) por meio da adaptação do modelo de grupoterapia cognitivo-comportamental a modalidade individual.

A avaliação de efetividade da TSCP-AS demonstrou que os profissionais apresentaram altos índices de impacto e transferência de aprendizagem. Estes achados sugerem que as estratégias utilizadas na TSCP-AS, tais como o desenvolvimento de uma capacitação estruturada (Grossman \& Salas, 2011; Rakovshik \& McManus, 2010), utilização de um manual para orientar a intervenção (Kendall \& Beidas, 2007) e realização de supervisões (Clarke, 2002; Rakovshik \& McManus, 2010), foram efetivas na promoção da qualificação profissional dos participantes.

Foi investigado se as relações do impacto e a transferência de aprendizagem poderiam compor uma espiral de crescimento, considerando que esses estão fortemente associados (Abbad et al., 2004). As relações entre os índices de impacto e transferência de aprendizagem evidenciaram a existência de uma espiral de crescimento, na qual os índices de impacto repercutem sobre a transferência de aprendizagem, assim como a relação inversa. Compreende-se que esta espiral de crescimento origina-se como uma consequência de uma relação cíclica, na qual os benefícios associados ao impacto (e.g. motivação, envolvimento com o trabalho, melhor desempenho laboral) promovem o aumento dos índices de transferência de aprendizagem (Cheng \& Ho, 2001; Pilati \& Abbad, 2005). Da mesma forma, os efeitos positivos da transferência de aprendizagem, tal como atendimento efetivo das demandas por meio das técnicas aprendidas, repercutem nos níveis de motivação e envolvimento dos(as) profissionais com o seu trabalho e, consequentemente, aumentam os níveis de impacto (Taxman et al., 2014; Zerbini \& Abbad, 2010).

\section{CONSIDERAÇÕES FINAIS}

As repercussões da TSCP-AS sobre o desenvolvimento do trabalho dos(as) participantes, identificadas nos altos índices de impacto e transferência de aprendizagem, sugerem que os conteúdos e técnicas trabalhadas na capacitação atenderam às demandas dos profissionais nesta temática. Esses resultados demonstram o potencial da TSCP-AS como uma ferramenta à qualificação dos profissionais de psicologia ao uso de tratamentos baseados em evidências. Além disso, estes achados apontam a relevância de serem desenvolvidas capacitações para instrumentalizar os profissionais de psicologia no atendimento de vítimas de abuso sexual, tendo em vista as lacunas presentes na formação desses profissionais (CFP, 2009; Freitas \& Habigzang, 2013).

O presente estudo contribui ao apresentar as evidências de efetividade da TSCP-AS como uma ferramenta à qualificação dos profissionais de psicologia que trabalham com temáticas relacionadas ao AS. 
Entretanto, este estudo apresenta algumas limitações. As limitações da amostra referem-se à participação predominantemente de profissionais do sexo feminino, além da TSCP-AS ter sido desenvolvida apenas no estado do Rio Grande do Sul, Brasil. A ausência de um estudo de follow-up para avaliar os níveis de impacto e transferência de aprendizagem da TSCP-AS em um terceiro momento constitui outra limitação deste estudo.
Sugere-se o desenvolvimento de estudos que avaliem efetividade TSCP-AS com amostras de diferentes localidades, assim como a inclusão de entrevistas de follow-up com os participantes que concluíram a capacitação. A replicação da pesquisa apresentada possibilitaria o desenvolvimento de evidências robustas da efetividade da TSCP-AS, assim como da compreensão dos processos de impacto e transferência de aprendizagem dos profissionais capacitados.

\section{REFERÊNCIAS}

Abbad, G. (1999). Um modelo integrado de avaliação do impacto do treinamento no trabalho - IMPACT. Tese de doutorado não-publicada, Curso de Pós-Graduação em Psicologia da Universidade de Brasília. Brasília.

Abbad, G. S., Borges-Andrade, J. E., \& Sallorenzo, L. H. (2004). Self-assesment of training impact at work: Validation of a measurement scale. Revista Interamericana de Psicologia, 38, 277-284. Recuperado de http://www.psicorip.org/ Resumos/PerP/RIP/RIP036a0/RIP03829.pdf

Abbad, G. S., Gama, A. L. G., \& Borges-Andrade, J. E. (2000). Treinamento: Análise do relacionamento da avaliação nos níveis de reação, aprendizagem e impacto no trabalho. Revista de Administração Contemporânea, 4(3), 25-45. http://dx.doi.org/10.1590/S1415-65552000000300003

Aguinis, H. \& Kraiger, K. (2009). Benefits of training and development for individuals and teams, organizations, and society. Annual Review of Psychology, 60, 451-474. http://dx.doi.org/10.1146/annurev.psych.60.110707.163505

Alaggia, R. (2005). Discloing the trauma of child sexual abuse: A gender analysis. Journal of Loss and Trauma, 10, 453-470. http://dx.doi.org/10.1080/15325020500193895

Brasil (1990). Diário Oficial da União. Estatuto da Criança e do Adolescente, Lei Federal no 8.069 , de 13 de julho de 1990, Brasília, DF.

Cheng, E. W. L., \& Ho, D. C. K. (2001). A review of transfer of training studies in the past decade. Personnel Review, 30(1), 102-118. http://dx.doi.org/10.1108/00483480110380163

Chen, L. P., Murad, M. H., Paras, M. L., Colbenson, K. M., Sattler, A. L., Goranson, E. N., Elamin, M. B., Seime, R. J., Shinozaki, G., \& Prokop, L. J. (2010). Sexual Abuse and Lifetime Diagnosis of psychiatric disorders: Systematic review and meta-analysis. Mayo Clinic Proceedings, 85, 618-629. http://dx.doi.org/10.4065/mcp.2009.0583

Clarke, N. (2002). Job/work environment factors influencing training transfer within a human service agency: some indicative support for Baldwin and Ford's transfer climate construct. International Journal of Training and Development, 6(3),146-162. http://dx.doi.org/10.1111/1468-2419.00156

Cohen, J. A., Deblinger, E., Mannarino, A. P., \& Steer, R. (2004). A Multisite randomized controlled trial for children with sexual abuse - related PTSD symptoms. Journal American Academy of Child and Adolescent Psychiatry, 43(4), 393-402. http://dx.doi.org/10.1097/01.chi.0000111364.94169.f9

Cohen, J. A., Mannarino, A. P., \& Knudsen, K. (2005). Treating sexually abused children: One year follow-up of a randomized controlled trial. Child Abuse \& Neglect, 29, 135-145. http://dx.doi.org/10.1016/j.chiabu.2004.12.00

Fergusson, D. M., Boden, J. M., \& Horwood, L. J. (2008). Exposure to childhood sexual and physical abuse and adjustment in early adulthood. Child Abuse \& Neglect, 32(6), 607-19. http://dx.doi.org/10.1016/j.chiabu.2006.12.018

Freitas, C. P. P. (2013). Avaliação de impacto de uma tecnologia social para profissionais de psicologia que trabalham com vítimas de violência sexual (Dissertação de mestrado). Recuperado de http://hdl.handle.net/10183/76520.

Freitas, C. P. P. \& Habigzang, L. F. (2013). Percepções de psicólogos sobre a capacitação para intervenção com vítimas de violência sexual. Psicologia Clínica, 25(2), 215-230. http://dx.doi.org/10.1590/S0103-56652013000200013

Freitas, C. P. P. \& Habigzang, L. F. (2014). Tecnologia Social de Capacitação Profissional para Intervenção com adolescentes vítimas de violência sexual. In L. F. Habigzang, E. Diniz, \& S. H. Koller (Eds.), Trabalhando com adolescentes: Teoria e Pesquisa (pp. 309-327). Porto Alegre: Artmed.

Garavaglia, P. (1995). Transfer of Training: Making Training Stick - Practical Guidelines for Training and development professionals. Alexandria: American Society for Training and Development.

Grossman, R. \& Salas, E. (2011). The transfer of training: What really matters. International Journal of Training and Development, 15(2), 103-120. http://dx.doi.org/10.1111/j.1468-2419.2011.00373.x

Habigzang, L. F. \& Koller, S. H. (Eds.) (2011). Intervenção psicológica para crianças e adolescentes vítimas de violência sexual: manual de capacitação para profissionais. Porto Alegre: Casa do Psicólogo.

Habigzang, L. F., Damásio, B. F., \& Koller, S. H. (2013). Impact evaluation of a cognitive behavioral group therapy model in Brazilian sexually abused girls. Journal of Child Sexual Abuse, 22, 173-190. http://dx.doi.org/10.1080/10538712. 2013.737445 
Habigzang, L. F., Ramos, M. S., \& Koller, S. H. (2011). A revelação de abuso sexual: As medidas adotadas pela rede de apoio. Psicologia: Teoria e Pesquisa, 27, 467-473. http://dx.doi.org/10.1590/S0102-37722005000300011

Habigzang, L. F., Stroeher, F., Hatzenberguer, R., Cunha, R. C., Ramos, M., \& Koller, S. H. (2009). Grupoterapia cognitivo-comportamental para crianças e adolescentes vítimas de abuso sexual. Revista de Saúde Pública, 43, 70-78. http://dx.doi.org/10.1590/S0034-89102009000800011

Haukoos, J. S. \& Lewis, R. J. (2005). Advanced statistics: Bootstrapping confidence intervals for statistics with "difficult" distributions. Academic Emergency Medicine, 12(4), 360-365. http://dx.doi.org/10.1197/j.aem.2004.11.018

Hetzel-Riggin, M. D., Brausch, A. M., \& Montgomery, B. S. (2007). A meta-analytic investigation of therapy modality outcomes for sexually abused children and adolescents: An exploratory study. Child Abuse \& Neglect, 31, 125-141. http://dx.doi.org/10.1016/j.chiabu.2006.10.007

Hohendorff, J. V. (2012). Adaptação e avaliação de uma intervenção cognitivo-comportamental para meninos vítimas de violência sexual (Dissertação de mestrado). Recuperado de http://www.lume.ufrgs.br/handle/10183/55080.

Hohendorff, J. V., Habigzang, L. F., \& Koller, S. H. (in press). Descrição e Processo de Adaptação de um Modelo de Terapia Cognitivo-Comportamental para Meninos Vítimas de Violência Sexual. In A. P. Serafim (Ed.), Temas em psiquiatria forense e psicologia jurídica III: Violência sexual. São Paulo: Vetor Editora.

Kendall, P. C. \& Beidas, R. S. (2007). Smothing the trial for dissemination of evidence-based practices for youth: Flexibility within fidelity. Professional Psychology Research and Practice, 38(1), 13-20. http://dx.doi.org/10.1037/07357028.38.1.1

Lisak, D. (1994). The psychological impact of sexual abuse: Content analysis of interviews with male survivors. Journal of Traumatic Stress, 7(4), 525-548. http://dx.doi.org/10.1002/jts.2490070403

Luna, G. L. M., Ferreira, R. C., \& Vieira, L. J. E. S. (2010). Notificação de maus-tratos em crianças e adolescentes por profissionais da Equipe Saúde da Família. Ciência e Saúde Coletiva, 15(2), 481-491. http://dx.doi.org/10.1590/ S1413-81232010000200025

Maniglio, R. (2009). The impact of child sexual abuse on health: A systematic review of reviews. Clinical Psychology Review, 29, 647-657. http://dx.doi.org/10.1016/j.cpr.2009.08.003

Pilati, R. \& Abbad, G. (2005). Análise fatorial confirmatória da escala de impacto do treinamento no trabalho. Psicologia: Teoria e Pesquisa, 21(1), 43-51. http://dx.doi.org/10.1590/S0102-37722005000100007

Rakovshik, S. G. \& McManus, F. (2010). Establishing evidence-based training in cognitive behavioral therapy: A review of current empirical findings and theoretical guidance. Clinical Psychology Review, 30, 496-516. http://dx.doi. org/10.1016/j.cpr.2010.03.004

Rodríguez, C. M. \& Gregory, S. (2005). Qualitative study of transfer of training of student employees in a service industry. Journal of Hospitality \& Tourism Research, 29(1), 42-66. http://dx.doi.org/10.1177/1096348004270753

Sanson, A. (2011). Building bridges between research and social policy: An australian perspective. International Society for the Study of Behavioural Development (ISSBD Bulettin), 60(2), 2-6. Recuperado de http://www.issbd.org/ resources/files/JBD_Nov11.pdf

Saywitz, K. J., Mannarino, A. P., Berliner, L., \& Cohen, J. A. (2000). Treatment for sexually abused children and adolescents. American Psychologist, 55, 1040-1049. http://dx.doi.org/10.1037/0003-066X.55.9.1040

Souza, L. K., McCarthy, S., \& Gauer, G. (2012). Teaching Psychology in South America. In S. McCarthy, K. L. Dickson, J. Cranney, A. Trapp, \& V. Karandashev (Eds.), Teaching Psychology around the World (Vol. 3, pp. 350-374). Newcastle: Cambridge Scholars Publishing.

Taxman, F. S., Henderson, C., Young, D., \& Farrell, J. (2014). The Impact of Training Interventions on Organizational Readiness to Support Innovations in Juvenile Justice Offices. Administration and Policy in Mental Health and Mental Health Services Research, 41, 177-188. http://dx.doi.org/10.1007/s10488-012-0445-5

World Health Organization. (2006). Preventing child maltreatment: A guide to taking action and generating evidence. Geneva: World Health Organization. Recuperado de http:/www.who.int/topics/child abuse/en/

Zerbini, T. \& Abbad, G. (2010). Qualificação profissional a distância: Avaliação da transferência de treinamento. Paidéia, 20(47), 313-323. http://dx.doi.org/10.1590/S0103-863X2010000300004

\footnotetext{
Autores: do Sul.

Endereço para correspondência:

Clarissa Pinto Pizarro de Freitas

Rua Ramiro Barcelos, 2600, sala 104

CEP 90035-003 Porto Alegre, RS, Brasil

E-mail: freitas.cpp@gmail.com
}

Clarissa Pinto PiZarro de Freitas - Doutoranda em Psicologia, Instituto de Psicologia, Universidade Federal do Rio Grande do Sul. Luísa Fernanda Habigzang - Doutora, Professora Associada no Instituto de Psicologia, Pontifícia Universidade Católica do Rio Grande

Sílvia Helena Koller - Doutora, Professora Associada no Instituto de Psicologia, Universidade Federal do Rio Grande do Sul. 